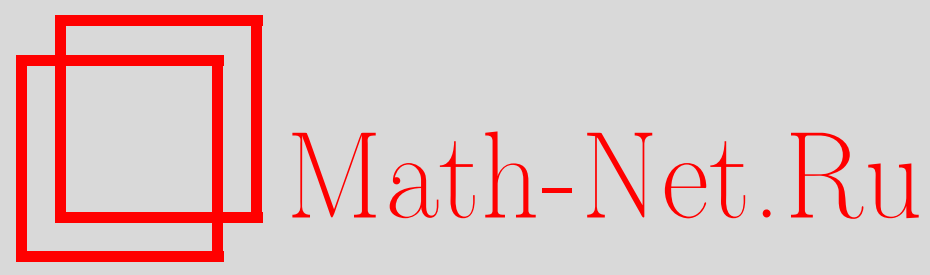

K. А. Попков, Диагностика состояний контактов, Дискрет. матем., 2013, том 25, выпуск 4, 30-40

DOI: https://doi.org/10.4213/dm1255

Использование Общероссийского математического портала Math-Net.Ru подразумевает, что вы прочитали и согласны с пользовательским соглашением http://www.mathnet.ru/rus/agreement

Параметры загрузки:

IP : 54.172 .240 .79

26 апреля 2023 г., 03:01:17 


\title{
Диагностика состояний контактов
}

\author{
() 2013 г. K. А. Попков
}

\begin{abstract}
В работе рассматривается задача распознавания состояний контактов с использованием экспериментов, заключающихся в составлении из заданных контактов произвольных двухполюсных контактных схем с последующим «прозваниванием» этих схем, т. е. нахождением булевых функций, реализуемых составляемыми схемами.
\end{abstract}

\section{Введение}

Описания физических принципов работы релейно-контактных схем и тех явлений, которые наблюдаются при работе таких схем, можно найти, например, в монографиях $[1,2]$. Суть общепринятой математической модели контактной схемы и тех элементов (т. е. контактов), из которых строятся эти схемы, с исчерпывающей полнотой и ясностью представлена в [3]; именно такая математическая модель является объектом исследования и рассматривается ниже.

Представим, что имеются $N$ контактов $(N \geqslant 1)$, занумерованных числами от 1 до $N$, из которых $N_{1}$ контактов с номерами от 1 до $N_{1}$ являются замыкающими, а $N_{2}$ контактов с номерами от $N_{1}+1$ до $N-$ размыкающими, где $N_{2}=N-N_{1} \quad\left(N_{1}\right.$ или $N_{2}$ могут быть равны 0$)$. В исправном состоянии каждый замыкающий контакт, рассматриваемый как простейшая контактная схема, реализует между своими концами (полюсами схемы) булеву функцию $x_{i}$, а размыкающий контакт - булеву функцию $\overline{x_{i}}$, где $x_{i}$ - отвечающая данному контакту переменная из множества $\left\{x_{1}, x_{2}, \ldots, x_{N}\right\}$. Число замыкающих контактов $\left(N_{1}\right)$, и соответственно, число размыкающих контактов $\left(N_{2}\right)$ предполагаются известными. В неисправном состоянии каждый контакт реализует между своими концами одну из булевых констант, то есть 0 (при обрыве контакта) или 1 (при замыкании контакта). Замыкание (или, как ещё иногда говорят, короткое замыкание) здесь рассматривается как разновидность неисправности контакта (а не как функционирование исправного контакта, т. е. изменение его проводимости с нулевой на единичную). Предполагается, что среди данных $N$ контактов не более $k$ контактов могут быть неисправны, где $k$ - заданное натуральное число, $k \leqslant N$. Можно составлять любые двухполюсные контактные схемы из данных контактов и наблюдать выдаваемые схемами значения на любых наборах значений переменных. При этом предполагается, что в каждой из построенных схем разным контактам могут отвечать одни и те же переменные из множества $\left\{x_{1}, x_{2}, \ldots, x_{N}\right\}$, а в разных схемах одному и тому же контакту могут отвечать разные переменные из этого же множества (таким образом, вовсе не обязательно, чтобы в каждой схеме контакту с номером $n$ отвечала переменная $\left.x_{n}\right)$. Например, в одной 
и той же схеме двум или более контактам может отвечать переменная $x_{1}$, а одному и тому же контакту в одной схеме может отвечать переменная $x_{1}$, в другой $-x_{2}$ и т.д.

Задача заключается в том, чтобы продиагностировать контакты, то есть определить состояние каждого (исправен или неисправен, если неисправен, то тип неисправности), используя при тестировании по возможности меньшее число схем.

Предполагается, что в процессе экспериментирования исправные контакты остаются исправными, неисправные контакты - неисправными и тип неисправности каждого неисправного контакта сохраняется.

\section{1. Основные определения и вспомогательные утвер- ждения}

Будем называть неисправностъю системы контактов любое множество неисправностей контактов при условии, что число этих неисправностей не больше $k$. (В частности, случай, когда все контакты исправны, является одним из видов неисправности системы контактов.)

Неисправность любого контакта можно представить в виде упорядоченной пары $\left\{n_{j}, \delta_{j}\right\}$, где $n_{j}$ - номер этого контакта, $\delta_{j}$ - булева константа, которую он реализует (между своими концами). Соответственно, любую неисправность системы контактов можно представить в виде множества $\left\{\left\{n_{1}, \delta_{1}\right\}, \ldots,\left\{n_{s}, \delta_{s}\right\}\right\}$, где $s$ - число неисправных контактов, $n_{1}, \ldots, n_{s}$ - номера неисправных контактов, $\delta_{j}$ - булева константа, которую реализует между своими концами контакт $n_{j}$.

Диагностическим тестом назовём такой набор двухполюсных контактных схем $S_{1}, \ldots, S_{l}$, составленных из заданных контактов, что для любых двух различных неисправностей системы контактов наборы реализуемых схемами функций не совпадают (т. е. для любых двух неисправностей существует такая схема $S_{i}$, что реализуемая этой схемой функция при первой неисправности не совпадает с реализуемой этой же схемой функцией при второй неисправности). Число $l$ назовём длиной этого теста.

Отметим, что данному определению диагностического теста равносильно следующее: диагностический тест - это такой набор двухполюсных контактных схем $S_{1}, \ldots, S_{l}$, составленных из заданных $N$ контактов, что по набору функций, реализуемых этими схемами, можно однозначно определить состояние каждого из $N$ контактов.

В качестве тривиального диагностического теста (длины $N$ ), очевидно, можно взять множество из $N$ контактных схем, каждая из которых представляет собой один из заданных контактов.

Отметим также следующее обстоятельство: если в исходной постановке задачи в качестве неисправностей контактов допускаются только обрывы контактов (или только их замыкания), т. е. неисправности контактов предполагаются однотипными, то для диагностики контактов достаточно одной схемы, состоящей из $N$ параллельно (соответственно, последовательно) соединённых заданных контактов, в 
которой контакту с номером $n, n=1, \ldots, N$, отвечает переменная $x_{n}$. Действительно, при любой неисправности системы контактов функция, реализуемая этой схемой, представляет собой дизъюнкцию (соответственно, конъюнкцию) переменных, отвечающих исправным замыкающим контактам, и отрицаний переменных, отвечающих исправным размыкающим контактам, откуда следует, что состояние каждого контакта определяется по этой функции однозначно. Ввиду тривиальности этого случая больше на нём останавливаться не будем.

Утверждение 1. Пусть набор контактных схем $S_{1}, \ldots, S_{l}$ является диагностическим тестом. Тогда существует такой набор контактных схем $S_{1}^{\prime}, \ldots, S_{l}^{\prime}$, также являющийся диагностическим тестом, что в каждой из схем $S_{1}^{\prime}, \ldots, S_{l}^{\prime}$ любым двум разным контактам отвечают разные переменные.

Доказательство. Рассмотрим любую схему $S_{i}$ из $\left\{S_{1}, \ldots, S_{l}\right\}$. Пусть в ней участвуют переменные $x_{i, 1}, x_{i, 2}, \ldots, x_{i, n_{i}}$. Определим множества $M_{i, j}, j=1,2, \ldots, n_{i}$, как множества всех контактов, которым в схеме $S_{i}$ отвечает переменная $x_{i, j}$. Перенумеруем отвечающие контактам в этой схеме переменные следующим образом. В каждом из множеств $M_{i, j}$ выделим по одному контакту и будем считать, что этому контакту по-прежнему соответствует переменная $x_{i, j}$. Каждому же контакту, кроме выделенных, поставим в соответствие некоторую переменную из числа $x_{1}, x_{2}, \ldots, x_{N}$ так, чтобы любым двум различным заданным контактам отвечали разные переменные (это можно сделать, так как в схеме $S_{i}$ участвуют не более $N$ контактов). Получим некоторую схему $S_{i}^{\prime}$, в которой любым двум разным контактам отвечают разные переменные (см. рис. 1). В итоге получим набор схем $S_{1}^{\prime}, \ldots, S_{l}^{\prime}$.
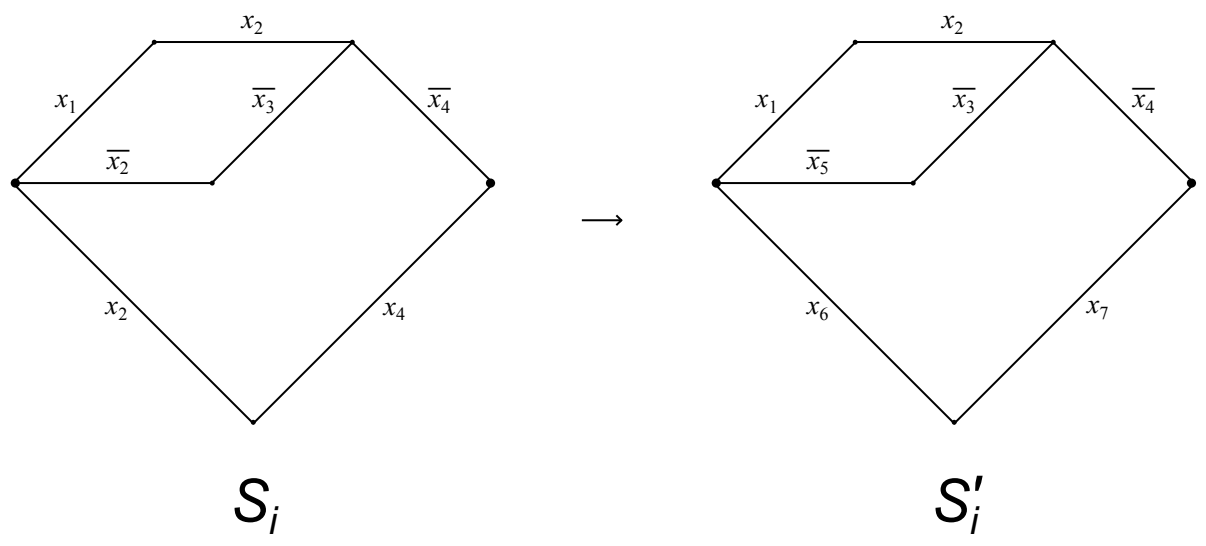

Рис. 1

Рассмотрим любые две различные неисправности системы контактов. Так как $\left\{S_{1}, \ldots, S_{l}\right\}$ - диагностический тест, то существует такая схема $S_{i}$, что функции, реализуемые схемой $S_{i}$ при этих неисправностях, не совпадают. Но если в схеме $S_{i}^{\prime}$ для любого $j$ от 1 до $n_{i}$ отождествить все переменные, отвечающие контактам из множества $M_{i, j}$, с переменной $x_{i, j}$, то схема $S_{i}^{\prime}$, очевидно, будет функционировать в точности как схема $S_{i}$ как при отсутствии неисправностей контактов, так и при их наличии. Таким образом, функции, реализуемые схемой $S_{i}^{\prime}$ при рассматриваемых 
двух неисправностях, будут совпадать с соответствующими функциями для схемы $S_{i}$ и, следовательно, различаться между собой. Однако эти функции получаются из исходных функций, реализуемых схемой $S_{i}^{\prime}$ при рассматриваемых двух неисправностях, путём отождествления части переменных; таким образом, схема $S_{i}^{\prime}$ реализует при этих неисправностях различные функции. Отсюда следует, что $\left\{S_{1}^{\prime}, \ldots, S_{l}^{\prime}\right\}-$ диагностический тест. Утверждение 1 доказано.

Введём функцию $L\left(N_{1}, N_{2}, k\right)$, равную длине самого короткого диагностического теста для $N_{1}$ замыкающих и $N_{2}$ размыкающих контактов, среди которых не более чем $k$ неисправных. Пусть $L(N, k)=L(N, 0, k)$.

Утверждение 2. Если $N_{1}+N_{2}=N$, mo $L\left(N_{1}, N_{2}, k\right)=L(N, 0, k)$.

Доказательство. В силу утверждения 1 для $N_{1}$ замыкающих и $N_{2}$ размыкающих контактов, среди которых не более чем $k$ неисправных, существует диагностический тест $\left\{S_{1}, \ldots, S_{l}\right\}$ длины $l=L\left(N_{1}, N_{2}, k\right)$, такой, что в каждой входящей в него схеме любым двум разным контактам отвечают разные переменные. В каждой из схем $S_{1}, \ldots, S_{l}$ заменим каждый размыкающий контакт на замыкающий контакт с тем же номером (переменные, отвечающие контактам, сохраняются; см. рис. 2). Получим набор схем $S_{1}^{\prime}, \ldots, S_{l}^{\prime}$. Покажем, что данный набор является диагностическим тестом для $N$ замыкающих контактов, среди которых не более чем $k$ неисправных.
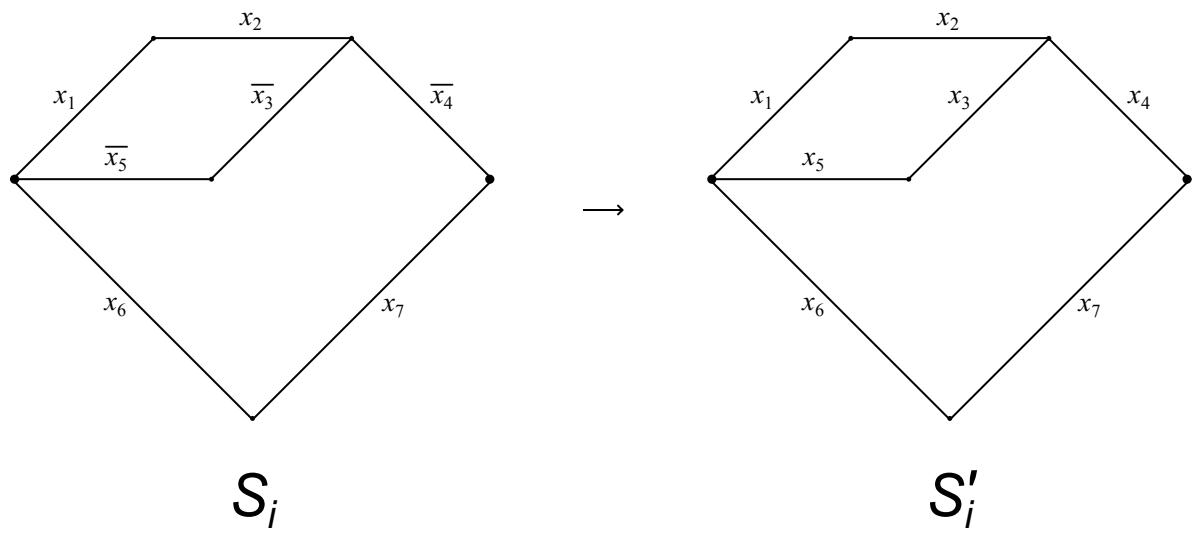

Рис. 2

Пусть $H_{1}^{\prime}$ и $H_{2}^{\prime}$ - две произвольные различные неисправности системы контактов для $N$ замыкающих контактов. Рассмотрим следующие две неисправности $H_{1}$ и $H_{2}$ системы контактов для $N_{1}$ замыкающих и $N_{2}$ размыкающих контактов: пусть при неисправности $H_{1}$ неисправны в точности контакты с теми же номерами, что и при $H_{1}^{\prime}$, и тип неисправности у контактов с одинаковыми номерами одинаковый; аналогично для $H_{2}$ и $H_{2}^{\prime}$. Так как $\left\{S_{1}, \ldots, S_{l}\right\}$ - диагностический тест, то существует такая схема $S_{i}$, что функции, реализуемые схемой $S_{i}$ при неисправностях $H_{1}$ и $H_{2}$ (обозначим их для краткости $f\left(S_{i}, H_{1}\right)$ и $f\left(S_{i}, H_{2}\right)$ ), не совпадают. Легко видеть, что функции, реализуемые схемой $S_{i}^{\prime}$ при неисправностях $H_{1}^{\prime}$ и $H_{2}^{\prime}$ (обозначим их $f\left(S_{i}^{\prime}, H_{1}^{\prime}\right)$ и $\left.f\left(S_{i}^{\prime}, H_{2}^{\prime}\right)\right)$, получаются из функций, соответственно, $f\left(S_{i}, H_{1}\right)$ 
и $f\left(S_{i}, H_{2}\right)$ заменой всех переменных, отвечающих в схеме $S_{i}$ размыкающим контактам, их отрицаниями. Отсюда и из того, что $f\left(S_{i}, H_{1}\right)$ не совпадает с $f\left(S_{i}, H_{2}\right)$, следует несовпадение функций $f\left(S_{i}^{\prime}, H_{1}^{\prime}\right)$ и $f\left(S_{i}^{\prime}, H_{2}^{\prime}\right)$. Это означает, что набор схем $S_{1}^{\prime}, \ldots, S_{l}^{\prime}$ является диагностическим тестом для $N$ замыкающих контактов, среди которых не более чем $k$ неисправных. Таким образом, $L(N, 0, k) \leqslant l=L\left(N_{1}, N_{2}, k\right)$. Аналогично можно показать, что $L\left(N_{1}, N_{2}, k\right) \leqslant L(N, 0, k)$. Утверждение 2 доказано.

Из утверждения 2 следует, что для нахождения $L\left(N_{1}, N_{2}, k\right)$ достаточно знать только $L\left(N_{1}+N_{2}, 0, k\right)=L(N, 0, k)=L(N, k)$. Поэтому далее без ограничения общности будем считать, что все заданные контакты замыкающие.

\section{2. Формулировка и доказательство основного ре- зультата}

Основным результатом данной работы является следующее утверждение

Теорема 1. Пусть $N \geqslant 36$ u $2 k\lceil\sqrt{k}\rceil \leqslant N$. Тогда $L(N, k) \leqslant k+1$.

Доказательство. Согласно постулату Бертрана (см., например, [4], стр. 30) для любого натурального $n>3$ на интервале $(n ; 2 n-2)$ содержится хотя бы одно простое число. Отсюда следует, что для любого (не обязательно целого) $x \geqslant 1$ на отрезке $[x ; 2 x]$ содержится хотя бы одно простое число. Действительно, если $1 \leqslant x \leqslant 2$, то $2 \in[x ; 2 x]$; если $2<x \leqslant 3$, то $3 \in[x ; 2 x]$. Если же $x>3$, то $\lceil x\rceil>3, x \leqslant\lceil x\rceil$ и $2\lceil x\rceil-2<2(x+1)-2=2 x$, откуда следует, что на интервале $(\lceil x\rceil ; 2\lceil x\rceil-2)$ содержится хотя бы одно простое число, а сам этот интервал содержится в отрезке $[x ; 2 x]$. Таким образом, на отрезке $[x ; 2 x]$ содержится хотя бы одно простое число.

Определим простое число $p$ следующим образом. Пусть $k<\sqrt{N}$. Тогда выполнено неравенство

$$
2\lceil\sqrt{k}\rceil \leqslant \sqrt{N}
$$

Действительно, если $k \leqslant 9$, то $2\lceil\sqrt{k}\rceil \leqslant 6 \leqslant \sqrt{N}$; если же $k>9$, то, как нетрудно убедиться, выполнено неравенство $2\lceil\sqrt{k}\rceil \leqslant k$, но $k<\sqrt{N}$.

Выберем в качестве $p$ любое простое число из отрезка $[\sqrt{N} ; 2 \sqrt{N}]$. Тогда $p \geqslant$ $\sqrt{N}>k$ и $p \leqslant 2 \sqrt{N}=\frac{2\lceil\sqrt{k}\rceil}{\sqrt{N}} \cdot \frac{N}{\lceil\sqrt{k}\rceil} \leqslant \frac{N}{\lceil\sqrt{k}\rceil}$ в силу (1).

Пусть теперь $k \geqslant \sqrt{N}$. Из условия теоремы следует, что

$$
2 k \leqslant \frac{N}{\lceil\sqrt{k}\rceil} .
$$

Выберем в качестве $p$ любое простое число из отрезка $[k ; 2 k]$. Тогда $p \geqslant k \geqslant \sqrt{N}$ и $p \leqslant 2 k \leqslant \frac{N}{\lceil\sqrt{k}\rceil}$ в силу $(2)$.

В обоих случаях получаем, что число $p$ удовлетворяет следующим неравенствам: $p \geqslant \sqrt{N}, p \geqslant k$ и $p \leqslant \frac{N}{\lceil\sqrt{k}\rceil}$.

Отождествим множество всех $N$ контактов с множеством $M=\{1, \ldots, N\}$ их номеров. Для выбранного $p$ построим множества $A_{i, j} \subset M, i=1, \ldots, k, j=1, \ldots, p$ : $A_{i, j}$ состоит из всех чисел из $M$, представимых в виде $p m+1+((j-1+m(i-$ 1) $\bmod p$ ), где $m$ - целое неотрицательное число, и множества $A_{k+1, j} \subset M, j=$ $1,2, \ldots,\left\lceil\frac{N}{p}\right\rceil: A_{k+1, j}$, состоит из всех чисел из $M$, представимых в виде $p(j-1)+t$, 
где $t$ - натуральное число от 1 до $p$. (Отметим, что $\frac{N}{p} \geqslant 1$, так как $p \leqslant \frac{N}{\lceil\sqrt{k}\rceil}$. Кроме того, $\left|A_{k+1, j}\right|=p$ для $j \leqslant\left\lfloor\frac{N}{p}\right\rfloor$; если $N$ не делится на $p$, то $\left|A_{k+1,\left\lceil\frac{N}{p}\right\rceil}\right|<p$.)

Пример 1. Вид множеств $A_{i, j}$ для $N=46, k=3, p=7$.

$$
\begin{array}{ll}
A_{1,1}=\{1,8,15,22,29,36,43\} & A_{2,1}=\{1,9,17,25,33,41\} \\
A_{1,2}=\{2,9,16,23,30,37,44\} & A_{2,2}=\{2,10,18,26,34,42,43\} \\
A_{1,3}=\{3,10,17,24,31,38,45\} & A_{2,3}=\{3,11,19,27,35,36,44\} \\
A_{1,4}=\{4,11,18,25,32,39,46\} & A_{2,4}=\{4,12,20,28,29,37,45\} \\
A_{1,5}=\{5,12,19,26,33,40\} & A_{2,5}=\{5,13,21,22,30,38,46\} \\
A_{1,6}=\{6,13,20,27,34,41\} & A_{2,6}=\{6,14,15,23,31,39\} \\
A_{1,7}=\{7,14,21,28,35,42\} & A_{2,7}=\{7,8,16,24,32,40\} \\
A_{3,1}=\{1,10,19,28,30,39\} & \\
A_{3,2}=\{2,11,20,22,31,40\} & A_{4,1}=\{1,2,3,4,5,6,7\} \\
A_{3,3}=\{3,12,21,23,32,41,43\} & A_{4,2}=\{8,9,10,11,12,13,14\} \\
A_{3,4}=\{4,13,15,24,33,42,44\} & A_{4,3}=\{15,16,17,18,19,20,21\} \\
A_{3,5}=\{5,14,16,25,34,36,45\} & A_{4,4}=\{22,23,24,25,26,27,28\} \\
A_{3,6}=\{6,8,17,26,35,37,46\} & A_{4,5}=\{29,30,31,32,33,34,35\} \\
A_{3,7}=\{7,9,18,27,29,38\} & A_{4,6}=\{36,37,38,39,40,41,42\}
\end{array}
$$

Введём функцию

$$
q(i)= \begin{cases}p & \text { при } i=1, \ldots, k, \\ \left\lceil\frac{N}{p}\right\rceil & \text { при } i=k+1,\end{cases}
$$

равную числу множеств $A_{i, j}$ для заданного $i$.

Лемма 1. Справедливы следующие утверждения:

1) Для любого $i$ от 1 до $k+1$ множества $A_{i, j_{1}}$ u $A_{i, j_{2}}$ не пересекаются, если $j_{1} \neq j_{2}$.

2) Для любого $i$ от 1 до $k+1$ выполняется соотношение $\bigcup_{j=1}^{q(i)} A_{i, j}=M$.

3) Для любых $i_{1}, i_{2}$ от 1 до $k+1, i_{2} \neq i_{2}, u$ любых $j_{1}, j_{2}$ множества $A_{i_{1}, j_{1}} u$ $A_{i_{2}, j_{2}}$ имеют не более одного общего элемента.

4) Для любых $i$ от 1 до $k$ и j от 1 до р множество $A_{i, j}$ содержит ровно $\left\lfloor\frac{N}{p}\right\rfloor$ чисел от 1 до $p\left\lfloor\frac{N}{p}\right\rfloor$

Справедливость утверждений 1) - 4) леммы 1 почти очевидным образом вытекает из геометрической интерпретации множеств $A_{i, j}$ как прямых или отрезков прямых в конечной аффинной плоскости порядка $p$ с $p^{2}$ точками (здесь существенно, что $p$ - простое число, $p \geqslant \sqrt{N}$ и $p \geqslant k$ ). Определение и свойства конечных плоскостей можно найти, например, в [5], гл. 1.

Перейдём к построению схем $S_{1}, S_{2}, \ldots, S_{k+1}$, которые составят диагностический тест. Для любого $i$ от 1 до $k+1$ построим схему $S_{i}$ следующим образом. Для каждого множества $A_{i, j}, j=1,2, \ldots, q(i)$, составим цепь из всех контактов, входящих 
в это множество, и поставим её в соответствие этому множеству (порядок контактов в цепи может быть произвольным). По утверждению 1) леммы 1 эти $p$ цепей попарно не пересекаются ни по одному контакту. Затем соединим все эти $q(i)$ цепей параллельно. Полюсами схемы объявим общие для всех цепей вершины.

Будем считать, что в каждой из построенных схем для любого $n$ от 1 до $N$ контакту с номером $n$ отвечает переменная $x_{n}$. Заметим, что по утверждению 2) леммы 1 в каждой из схем $S_{1}, S_{2}, \ldots, S_{k+1}$ содержатся все $N$ контактов. Кроме того, по построению выполнено следующее утверждение (обозначим его $(*))$ : каждому множеству $A_{i, j}, i=1,2, \ldots, k+1, j=1,2, \ldots, q(i)$, соответствует какая-то цепь в схеме $S_{i}$, и наоборот.

Идея дальнейших рассуждений состоит в следующем. При любой неисправности системы контактов среди схем $S_{1}, S_{2}, \ldots, S_{k+1}$ можно выделить "достаточно много" схем, которые не содержат цепей между полюсами, целиком состоящих из неисправных замкнутых контактов, и, соответственно, реализуют функции, отличные от тождественной единицы. Можно показать, что для любого исправного или неисправного замкнутого контакта хотя бы в одной схеме из числа выделенных схем в цепи, содержащей этот контакт, ни один контакт не оборван и хотя бы один контакт исправен. Далее уже нетрудно получить критерии того, что произвольный заданный контакт исправен, замкнут или оборван.

Отметим, что при фиксированной неисправности системы контактов любая из схем $S_{1}, S_{2}, \ldots, S_{k+1}$ реализует тождественную единицу тогда и только тогда, когда в ней содержится цепь между полюсами, целиком состоящая из неисправных замкнутых контактов. Действительно, если бы такой цепи не было, то при подаче вместо всех переменных схемы нулей все исправные контакты были бы разомкнуты и проводимость схемы была бы нулевой.

Введём обозначение $m=\left\lfloor\frac{N}{p}\right\rfloor$.

Лемма 2. Пусть при некоторой неисправности системы контактов среди контактов с номерами от 1 до рт ровно $r^{\prime}$ замкнутых. Тогда не более $r^{\prime}$ схем из числа $S_{1}, S_{2}, \ldots, S_{k}$ реализуют тождественную единицу.

Доказательство. При $r^{\prime}=k$ справедливость леммы очевидна. Пусть $r^{\prime}<k$. Предположим, что утверждение леммы не выполнено, т. е. некоторые схемы $S_{i_{1}}, S_{i_{2}}, \ldots, S_{i_{r^{\prime}+1}}$ реализуют тождественную единицу, где $i_{1}, i_{2}, \ldots, i_{r^{\prime}+1}$ - попарно различные индексы от 1 до $k$. Выберем из них произвольную схему $S_{i_{t}}, t \in$ $\left\{1, \ldots, r^{\prime}+1\right\}$. Так как она реализует тождественную единицу, то в ней существует цепь, состоящая целиком из неисправных замкнутых контактов. Этой цепи в силу утверждения $(*)$ соответствует некоторое множество $A_{i_{t}, j_{t}}$, которое также целиком состоит из неисправных замкнутых контактов. В нём определим подмножество $A_{i_{t}, j_{t}}^{\prime}$, содержащее в точности все контакты из $A_{i_{t}, j_{t}}$ с номерами от 1 до $p m$. Тогда все контакты в $A_{i_{t}, j_{t}}^{\prime}$ замкнуты и $\left|A_{i_{t}, j_{t}}^{\prime}\right|=m$ по утверждению 4) леммы 1. Отметим, что $m=\left\lfloor\frac{N}{p}\right\rfloor \geqslant\lceil\sqrt{k}\rceil$ в силу того, что $p \leqslant \frac{N}{\lceil\sqrt{k}\rceil}$. Так как $\lceil\sqrt{k}\rceil \geqslant 1$, то в $A_{i_{t}, j_{t}}^{\prime}$ содержится хотя бы один замкнутый контакт, откуда следует, что $r^{\prime} \geqslant 1$.

Так как $t$ - произвольный индекс от 1 до $r^{\prime}+1$, то определены множества $A_{i_{1}, j_{1}}^{\prime}, A_{i_{2}, j_{2}}^{\prime}, \ldots, A_{i_{r^{\prime}+1}, j_{r^{\prime}+1}}^{\prime}$. Пусть $n_{1}, n_{2}, \ldots, n_{r^{\prime}}$ - номера всех замкнутых контактов среди контактов с номерами от 1 до $\mathrm{pm}$. Очевидно, что каждый контакт в каждом из множеств $A_{i_{1}, j_{1}}^{\prime}, A_{i_{2}, j_{2}}^{\prime}, \ldots, A_{i_{r^{\prime}+1}, j_{r^{\prime}+1}}^{\prime}$ имеет один из номеров $n_{1}, n_{2}, \ldots, n_{r^{\prime}}$. Пусть контакт с номером $n_{s}, s=1, \ldots, r^{\prime}$, принадлежит ровно $\varphi(s)$ множествам из 
числа $A_{i_{1}, j_{1}}^{\prime}, A_{i_{2}, j_{2}}^{\prime}, \ldots, A_{i_{r^{\prime}+1}, j_{r^{\prime}+1}}^{\prime}$. Пусть

$$
\chi_{n}(A)= \begin{cases}1, & \text { если множество } A \text { содержит контакт с номером } n, \\ 0 & \text { в противном случае. }\end{cases}
$$

Легко видеть, что

$$
\sum_{s=1}^{r^{\prime}} \varphi(s)=\sum_{s=1}^{r^{\prime}} \sum_{t=1}^{r^{\prime}+1} \chi_{n_{s}}\left(A_{i_{t}, j_{t}}^{\prime}\right)=\sum_{t=1}^{r^{\prime}+1} \sum_{s=1}^{r^{\prime}} \chi_{n_{s}}\left(A_{i_{t}, j_{t}}^{\prime}\right)=\sum_{t=1}^{r^{\prime}+1}\left|A_{i_{t}, j_{t}}^{\prime}\right|=m\left(r^{\prime}+1\right),
$$

откуда следует, что хотя бы для одного $s$ выполняется соотношение

$$
\varphi(s) \geqslant\left\lceil\frac{m\left(r^{\prime}+1\right)}{r^{\prime}}\right\rceil=m+\left\lceil\frac{m}{r^{\prime}}\right\rceil \geqslant m+1,
$$

т. е. контакт с номером $n_{s}$ принадлежит по крайней мере $m+1$ множествам из числа $A_{i_{1}, j_{1}}^{\prime}, A_{i_{2}, j_{2}}^{\prime}, \ldots, A_{i_{r^{\prime}+1}, j_{r^{\prime}+1}}^{\prime}$. Обозначим эти $m+1$ множеств для краткости через $A_{1}^{\prime}, A_{2}^{\prime}, \ldots, A_{m+1}^{\prime}$, а контакт с номером $n_{s}$ через $K$. По построению каждое из этих множеств содержит ровно $m$ замкнутых контактов с номерами от 1 до $p m$, одним из которых является $K$. Так как индексы $i_{1}, i_{2}, \ldots, i_{r^{\prime}+1}$ попарно различны, то по утверждению 3) леммы 1 любые два из множеств $A_{i_{1}, j_{1}}^{\prime}, A_{i_{2}, j_{2}}^{\prime}, \ldots, A_{i_{r^{\prime}+1}, j_{r^{\prime}+1}}^{\prime}$, в том числе любые два из множеств $A_{1}^{\prime}, A_{2}^{\prime}, \ldots, A_{m+1}^{\prime}$, пересекаются не более чем по одному контакту. Но по построению контакт $K$ лежит в каждом из множеств $A_{1}^{\prime}, A_{2}^{\prime}, \ldots, A_{m+1}^{\prime}$. Отсюда следует, что множества $A_{1}^{\prime}, A_{2}^{\prime} \backslash\{K\}, A_{3}^{\prime} \backslash\{K\}, \ldots, A_{m+1}^{\prime} \backslash$ $\{K\}$ попарно не пересекаются. В объединении этих множеств лежат

$\left|A_{1}^{\prime}\right|+\left|A_{2}^{\prime}\right|-1+\left|A_{3}^{\prime}\right|-1+\ldots+\left|A_{m+1}^{\prime}\right|-1=m+m(m-1)=m^{2} \geqslant(\lceil\sqrt{k}\rceil)^{2} \geqslant k>r^{\prime}$

замкнутых контактов с номерами от 1 до $\mathrm{pm}$. С другой стороны, общее число замкнутых контактов с такими номерами равно $r^{\prime}$. Полученное противоречие доказывает лемму 2 .

Лемма 3. Пусть при некоторой неисправности системь контактов замкнуто ровно $r$ контактов. Тогда не более $r$ схем из числа $S_{1}, S_{2}, \ldots, S_{k+1}$ реализуют тождественную единицу.

Доказательство. Рассмотрим 2 случая.

1. Пусть замкнут хотя бы один контакт с номером, большим $p m$. Тогда $r \geqslant 1$ и среди контактов с номерами от 1 до $p m$ замкнуто не более $r-1$ контактов. В силу леммы 2 не более $r-1$ схем из числа $S_{1}, S_{2}, \ldots, S_{k}$ реализуют тождественную единицу. Тогда вне зависимости от того, какую функцию реализует схема $S_{k+1}$, не более $r$ схем из числа $S_{1}, S_{2}, \ldots, S_{k+1}$ реализуют тождественную единицу, что и требовалось доказать.

2. Пусть все замкнутые контакты имеют номера от 1 до $p m$. В силу леммы 2 не более $r$ схем из числа $S_{1}, S_{2}, \ldots, S_{k}$ реализуют тождественную единицу. Если схема $S_{k+1}$ реализует функцию, отличную от тождественной единицы, то утверждение леммы очевидно. Пусть схема $S_{k+1}$ реализует тождественную единицу. Тогда в ней содержится цепь, целиком состоящая из неисправных замкнутых контактов. Этой цепи в силу утверждения $\left(^{*}\right)$ соответствует некоторое множество $A_{k+1, j}$, состоящее 
целиком из неисправных замкнутых контактов. При этом, так как среди контактов с номерами, большими $p m$, нет ни одного замкнутого, $j \leqslant m$. Из построения множеств $A_{k+1, j}$ видно, что $\left|A_{k+1, j}\right|=p$ при $j \leqslant m$. С другой стороны, в силу неравенств $r \leqslant k \leqslant p$ множество $A_{k+1, j}$ может содержать $p$ замкнутых контактов только в том случае, когда $r=k=p$. Но тогда в этом множестве содержатся все неисправные контакты при рассматриваемой неисправности системы контактов. Так как $r=k$, то для доказательства леммы достаточно доказать, что хотя бы одна из схем $S_{1}, \ldots, S_{k}$, например, $S_{1}$, реализует функцию, отличную от тождественной единицы.

Предположим, что это не так. Тогда в схеме $S_{1}$ содержится цепь, состоящая целиком из неисправных замкнутых контактов, а значит, какое-то множество $A_{1, j^{\prime}}$ состоит целиком из неисправных замкнутых контактов. По утверждению 3) леммы 1 множества $A_{1, j^{\prime}}$ и $A_{k+1, j}$ пересекаются не более чем по одному контакту, а по утверждению 4) этой же леммы $\left|A_{1, j}\right| \geqslant\left\lfloor\frac{N}{p}\right\rfloor=m$. Но $m=\lceil\sqrt{k}\rceil=\lceil\sqrt{p}\rceil \geqslant\lceil\sqrt{2}\rceil=2$, поэтому $\left|A_{1, j^{\prime}}\right| \geqslant 2$. Значит, $A_{1, j^{\prime}}$ содержит хотя бы один контакт, не принадлежащий $A_{k+1, j}$ и, следовательно, исправный. Противоречие. Лемма 3 доказана.

Вернёмся к доказательству теоремы. Докажем, что построенный набор схем $S_{1}, S_{2}, \ldots, S_{k+1}$ является диагностическим тестом.

Зафиксируем некоторую неисправность системы контактов. Пусть при ней замкнуто ровно $r$ контактов. Возьмём контакт с произвольным номером $n$ от 1 до $N$ и обозначим его через $K$. Из леммы 3 следует, что из схем $S_{1}, S_{2}, \ldots, S_{k+1}$ можно выбрать $k+1-r$ схем, имеющих функцию проводимости, отличную от тождественной единицы. Обозначим эти схемы $S_{i_{1}}, \ldots, S_{i_{k+1-r}}$. Возьмём из них произвольную схему $S_{i_{t}}$. По утверждению 2) леммы 1 имеем $K \in \bigcup_{j=1}^{q\left(i_{t}\right)} A_{i_{t}, j}$, т. е. $K$ принадлежит какому-то $A_{i_{t}, j_{t}}$, где $j_{t}$ - натуральное число от 1 до $q\left(i_{t}\right)$. Таким образом, определены множества $A_{i_{1}, j_{1}}, A_{i_{2}, j_{2}}, \ldots, A_{i_{k+1-r}, j_{k+1-r}}$. Согласно утверждению 3) леммы 1 любые два из этих множеств пересекаются не более чем по одному контакту. Но по построению они пересекаются по контакту $K$. Значит, множества $A_{i_{1}, j_{1}} \backslash\{K\}, A_{i_{2}, j_{2}} \backslash\{K\}, \ldots, A_{i_{k+1-r}, j_{k+1-r}} \backslash\{K\}$ попарно не пересекаются. Число этих множеств равно $k+1-r$. С другой стороны, поскольку $r$ контактов замкнуты, то оборваны не более $k-r$ контактов. Таким образом, хотя бы в одном из этих множеств ни один контакт не оборван. Если теперь к этому множеству добавим контакт $K$, то получим одно из множеств $A_{i_{1}, j_{1}}, A_{i_{2}, j_{2}}, \ldots, A_{i_{k+1-r}, j_{k+1-r}}$, в котором ни один контакт, кроме, быть может, $K$, не оборван. Этому множеству в силу утверждения $(*)$ соответствует некоторая цепь $C$ из контактов в какой-то из схем $S_{i_{1}}, \ldots, S_{i_{k+1-r}}$ (обозначим эту схему через $S$ ), причём $K$ принадлежит $C$ и всякий отличный от $K$ контакт в этой цепи либо исправен, либо замкнут. Заметим, что в любой цепи между полюсами схемы $S$ присутствует хотя бы один незамкнутый контакт, так как иначе эта схема обладала бы тождественно единичной проводимостью, что неверно в силу выбора схем $S_{i_{1}}, \ldots, S_{i_{k+1-r}}$ и, в частности, схемы $S$. Предположим, что вместо всех переменных, отвечающих контактам, не входящим в цепь $C$, поданы нули. Тогда очевидно, что все остальные цепи между полюсами этой схемы будут обладать тождественно нулевой проводимостью. Значит, функция проводимости всей схемы будет равна функции проводимости цепи $C$, т. е. конъюнкции переменных, отвечающих исправным (незамкнутым) контактам в этой цепи, если контакт $K$ не оборван, либо равна тождественному нулю, если он оборван. Если контакт $K$ (имеющий, напомним, номер $n$ ) исправен, то эта функция существенно зависит от переменной 
$x_{n}$. Отсюда получаем, что и исходная функция, реализуемая схемой $S$ (до подачи вместо части переменных нулей), существенно зависит от $x_{n}$. Итак, если контакт $K$ исправен, то среди схем $S_{1}, \ldots, S_{k+1}$ хотя бы одна реализует функцию, существенно зависящую от $x_{n}$. Если же контакт $K$ неисправен, то очевидно, что ни одна из схем $S_{1}, \ldots, S_{k+1}$ не обладает этим свойством. Таким образом, по набору функций, реализуемых схемами $S_{1}, \ldots, S_{k+1}$, можно определить, исправен контакт $K$ или нет.

Далее, если контакт $K$ неисправен и при этом замкнут, то в цепи $C$ есть хотя бы один исправный контакт, и функция, реализуемая схемой $S$, существенно зависит от переменной, отвечающей этому контакту. Следовательно, в этом случае среди схем $S_{1}, \ldots, S_{k+1}$ можно выделить такую схему и такой контакт, находящийся в этой схеме в одной цепи с $K$, что функция проводимости этой схемы существенно зависит от переменной, отвечающей выделенному контакту. Если же $K$ неисправен и при этом оборван, то очевидно, что для любой схемы из числа $S_{1}, \ldots, S_{k+1}$ и любого контакта $K^{\prime}$, находящегося в этой схеме в одной цепи с $K$, функция проводимости этой схемы не зависит существенно от переменной, отвечающей выбранному контакту $K^{\prime}$ (так как проводимость этой цепи тождественно нулевая). Таким образом, зная, что контакт $K$ неисправен, по набору функций, реализуемых схемами $S_{1}, \ldots, S_{k+1}$, можно определить, замкнут он или оборван. В результате указанной процедуры состояние контакта $K$ определяется однозначно. Но в качестве $K$ можно взять любой контакт. Следовательно, по набору функций, реализуемых схемами $S_{1}, \ldots, S_{k+1}$, можно однозначно определить состояние каждого из $N$ контактов.

Теорема доказана.

Замечание 1. Можно проверить, что формулировка основной теоремы может быть усилена до следующей:

Пусть $k\left\lceil\frac{\sqrt{4 k+5}-1}{2}\right\rceil \leqslant N$ и на отрезке $\left[k ; \frac{N}{\left|\frac{\sqrt{4 k+5}-1}{2}\right|}\right]$ содержится хотя бы одно натуральное число, являющееся степенью простого числа. Тогда $L(N, k) \leqslant k+1$.

Нетрудно убедиться, что в такой формулировке теорема остаётся верна для всех пар $(N, k)$, для которых она верна в первоначальной формулировке, а также становится верна для некоторых новых пар (например, когда $N \leqslant 35, k$ мало). Улучшение достигается за счёт более точного выбора числа $p$ и уточнения оценок, используемых в доказательстве леммы 2. При этом число $p$ может не быть простым, но быть степенью простого числа. Для таких $p$ также существуют конечные аффинные плоскости порядка $p$, например, аффинные плоскости, получающиеся удалением одной прямой (вместе со всеми её точками) из дезарговой проективной плоскости порядка $p$ (см. [5], $\S \S 1.1-1.8,1.10 ;[6], \S \S 12.1-12.3)$. Случай $N=5, k=2$ в усиленной формулировке теоремы надо рассматривать отдельно, так как в этом случае не удаётся выбрать число такое $p$ на отрезке $\left[k ; \frac{N}{\left\lceil\frac{\sqrt{4 k+5}-1}{2}\right\rceil}\right]$, что $p \geqslant \sqrt{N}$.

В заключение автор выражает глубокую благодарность своему научному руководителю, профессору Н. П. Редькину, за постановку задачи и внимание к работе.

\section{Список литературы}

1. Гаврилов М. А., Теория релейно-контактных схем, Москва-Ленинград, 1950. 
2. Колдуэлл С., Логический синтез релейных устройств, Москва, 1962.

3. Лупанов О. Б., Асимптотические оченки сложности управляющих систем. Изд-во МГУ, Москва, 1984.

4. Галочкин А. И., Нестеренко Ю. В., Шидловский А. Б., Введение в теорию чисел. Издво МГУ, Москва, 1984.

5. Картеси Ф., Введение в конечные геометрии. Наука, Москва, 1980.

6. Холл М., Комбинаторика. Мир, Москва, 1970.

Статья поступила 16.05.2013. 